\title{
Superresolution in problems of remote sensing
}

\author{
Boris Lagovsky ${ }^{1, *}$, Alexander Samokhin ${ }^{1}$, and Malay Tripathy ${ }^{2}$ \\ ${ }^{1}$ Russian Technological University (MIREA), 78, Vernadsky av., Moscow, Russia \\ ${ }^{2}$ Amity University, Noida - 201313, Uttar Pradesh, India
}

\begin{abstract}
The analytical and numerical results of the development of new methods for obtaining the angular superresolution using partially known information about the source of signals are presented. Created high-speed algorithms allow you to use them in real time. The stability of the solutions obtained ensures adequate restoration of the radio images of objects. The noise immunity of the solutions obtained is higher than that of the known methods.
\end{abstract}

\section{Introduction}

One of the main directions of improvement of radar systems and remote sensing is to increase their informativeness. In turn, increasing the angular resolution of systems and their accuracy is one of the important components of this process. Angular superresolution can detect the location of small sources, detail image of the objects, specify the boundaries of the objects and so on.

The investigated problems of obtaining super-resolution belong to the class of inverse problems. They are ill-posed, because do not satisfy the second and third requirements for the correctness of the Hadamard problem. Attempts to increase the angular resolution compared to the Rayleigh criterion due to digital signal processing often lead to instabilities in solutions. They result in significant errors in solutions.

Solutions of such problems are especially sensitive to random components in the original data. When obtaining a solution with an angular resolution not exceeding the Rayleigh criterion, their effect may be negligible. However, when trying to get a higher resolution, the role of random components increases exponentially.

There are many known methods for achieving superresolution [1-4]. It should be noted that these methods are not universal and not always effective. Their effectiveness depends primarily on the signal-to-noise $(\mathrm{S} / \mathrm{N})$ ratio in the received signal. Basically, the mentioned signal processing methods work successfully and allow to increase the effective angular resolution several times with a SNR not lower than $20 \mathrm{~dB}$. To further increase the resolution twice, an order of magnitude increase in the SNR is required.

In [5-11], the efficiency of using algebraic methods for solving inverse problems described by Fredholm integral equations is shown. The use of these methods makes it possible to form radio images of objects with superresolution at low signal-to-noise ratios, or, in an alternative interpretation, to increase the range of remote sensing systems.

\footnotetext{
* Corresponding author: Robertlag@yandex.ru
} 


\section{Formulation of the problem}

Suppose that in the scan sector there is an object under investigation with finite angular dimensions. The received signal in the form of the envelope $U(\varphi, \theta)$,obtained by scanning the sector of view is a convolution:

$$
U(\varphi, \theta)=\int_{\Omega} f(\varphi-\alpha, \theta-\gamma) I(\alpha, \gamma) \cos \gamma d \alpha d \gamma
$$

where $f(\theta, \varphi)$ is the directional pattern (DP) of the antenna system, $\Omega$ is the region in which the radiation sources are located; $I(\varphi, \theta)$ is the unknown angular distribution of the amplitude of the reflected (or emitted) signal source.

The numerical problem of detailed image reconstruction of the source $I(\varphi, \theta)$ with the highest possible resolution based on the analysis of the received signal and the known RP is posed. Mathematically, the problem reduces to an approximate solution of the one- and two-dimensional Fredholm integral equation (IE) of the first kind of convolution type (1).

This problem is an inverse problem. Of the three conditions for the correctness of the stated problem according to Hadamard (existence, uniqueness, and stability of the solution), in the considered problems the second and third are violated. Due to the incorrectness of the problem, attempts to increase the resolution in comparison with the classical value lead, in general, to the appearance of instabilities in the solutions.

The algebraic methods used to solve the problem are based on the parametrization of one- and two-dimensional inverse problems. Solutions are represented in the form of expansions in prescribed sequences of functions $g_{m}(\varphi, \theta), m=1,2 \ldots$ orthogonal in sector $\Omega$ with unknown coefficients of decomposition $b_{m}$. Approximate solutions are expressed as a finite sum of $\mathrm{N}$ terms:

$$
I(\alpha, \varphi) \cong \sum_{m=1}^{N} b_{m} g_{m}(\alpha, \varphi)
$$

The coefficients $b_{m}$, providing the best root-mean-square approximation (2) to the true source, are found by solving a system of linear algebraic equations (SLAE).

$$
\mathrm{V}=\mathrm{G} \mathrm{B},
$$

where the unknown coefficients $b_{\mathrm{m}}$ form the vector B.

The functions are introduced

$$
\begin{gathered}
\chi_{m}(\varphi, \theta)=\int_{\Omega} f\left(\varphi-\varphi^{\prime}, \theta-\theta^{\prime}\right) g_{m}\left(\varphi^{\prime}, \theta^{\prime}\right) d \varphi^{\prime} d \theta^{\prime} \\
V_{m}=\int_{\Theta} U(\varphi, \theta) \chi_{m}(\varphi, \theta) d \varphi d \theta, \quad G_{j, m}=\int_{\Theta} \chi_{j}(\varphi, \theta) \chi_{m}(\varphi, \theta) d \varphi d \theta
\end{gathered}
$$

where $\Theta$ is the signal receiving sector, $(\varphi, \theta) \in \Theta \geq \Omega$.

The most stable solutions are obtained if the functions $\chi_{m}(\varphi, \theta)$ are orthogonal. In this case, only the elements on the main diagonal of the matrix $\mathrm{G}$ are different from zero. Then the coefficients $b_{m}$ are not found when solving ill-conditioned systems (3-5), but directly from each of the equations of system (3):

$$
\int_{\Theta} U(\varphi, \theta) \chi_{m}(\varphi, \theta) d \varphi d \theta=b_{m} \int_{\Theta} \chi_{m}(\varphi, \theta)^{2} d \varphi d \theta, \quad m=1,2 \ldots . N
$$


As orthogonal functions $\chi_{m}(\alpha)$, eigenfunctions of an IE (1) can be used. However, their numerical search, even in the case of a degenerate nucleus, is reduced to solving illconditioned problems. This leads to unstable inadequate solutions.

The question arises about the possibility and method of creating such a system of orthogonal functions $\tilde{g}_{m}(\varphi)$ in the area $\Omega$, the images of which $\tilde{\chi}_{m}(\varphi)$ are orthogonal in $\Theta$. Then it will be possible to use systems of functions simultaneously representing the solution and the signal $U$ in the form of expansions in systems of orthogonal functions.

This will increase the stability of numerical solutions of inverse problems. Solutions based on such systems of functions are especially effective at high noise levels.

To simplify the expressions obtained, we consider the one-dimensional case. First, we introduce the system of functions $g_{m}(\varphi), m=1,2 \ldots N$, which is orthonormal in $\Omega$. On its basis, in accordance with Eq. (4), we obtain a system of functions $\chi_{m}(\varphi)$, non-orthogonal in the area $\Theta$. Next, create a matrix of scalar products:

$$
S_{m, n}=\left(\chi_{m}, \chi_{n}\right)=\int_{\Theta} \chi_{m}(\varphi) \chi_{n}(\varphi) d \phi
$$

and through transformation

$$
\overline{\mathrm{S}}=\mathrm{T}^{\mathrm{T}} \mathrm{S} \mathrm{T},
$$

where the columns of the matrix $\mathrm{T}$ are the eigenvectors of the matrix $\mathrm{S}$ and $\mathrm{T}^{\mathrm{T}}$ is the transposed matrix we get the matrix in diagonal form. Using the found matrix $\mathrm{T}$, we introduce a new system of functions $\tilde{\chi}_{m}(\varphi)$ :

$$
\overline{\tilde{\chi}}_{m}(\varphi)=\sum_{j=1}^{N} T_{j, m} \chi_{j}(\varphi), \quad m=1,2 \ldots N
$$

The resulting system of functions (9) turns out to be orthogonal in $\Theta$, which is easily verified by direct calculation of scalar products.

Next, we find a system of functions $\widetilde{g}_{m}(\varphi)$ that generates an orthogonal system (9):

$$
\tilde{\chi}_{m}(\varphi)=\int_{\Omega} f\left(\varphi-\varphi^{\prime}\right) \widetilde{g}\left(\varphi^{\prime}\right) d \varphi^{\prime}, \quad m=1,2 \ldots N
$$

From Eq. (9) it follows:

$$
\tilde{\chi}_{m}(\varphi)=\sum_{j=1}^{N} T_{m, j} \int_{\Omega} f\left(\varphi-\varphi^{\prime}\right) g_{j}\left(\varphi^{\prime}\right) d \varphi^{\prime}=\int_{\Omega} f\left(\varphi-\varphi^{\prime}\right)\left(\sum_{j=1}^{N} T_{m, j} g_{j}\left(\varphi^{\prime}\right)\right) d \varphi^{\prime}
$$

Comparing Eq. (9) and Eq. (11):

$$
\widetilde{g}_{m}(\varphi)=\sum_{j=1}^{N} T_{m, j} g_{j}(\varphi)
$$

The found system of functions (12) turns out to be orthogonal in $\Omega$. Indeed, since the functions $g_{m}(\varphi)$ are orthogonal and the eigenvectors of the matrix $\mathrm{S}$ forming the matrix $\mathrm{T}$ are also orthogonal,

$$
\int_{\Omega} \widetilde{g}_{m}(\phi) \widetilde{g}_{n}(\phi) d \phi=\sum_{j, i=1}^{N} T_{m, j} T_{n, i} \int_{\Omega} g_{j}(\phi) g_{i}(\phi) d \phi=\left\{\begin{array}{l}
0, m \neq n \\
\lambda_{m}, m=n,
\end{array} \quad \lambda_{m}=\sum_{j=1}^{N} T^{2}{ }_{m, j}\right.
$$


As a result, a new orthogonal system of functions in $\Omega$ is constructed on the basis of a given system of $N$ orthogonal functions $\widetilde{g}_{m}(\varphi)$. This system should now be used in Eq. (2). It generates a system of functions $\tilde{\chi}_{m}(\varphi)$, orthogonal in the region $\Theta$ and allows to calculate the coefficients $b_{m}$ from Eq. (2) in accordance with Eq. (6).

The question of the choice of the initial system of functions $g_{m}(\varphi)$ remains open. With the same stability of algorithms based on different $g_{m}(\varphi)$, the error in the solution depends on the system of functions used.

The choice of the system $g_{m}(\varphi)$ should be made on the basis of a priori information about the solution.

If it is known in advance, for example, that an object has one or several sources with small angular size, then as $g_{m}(\varphi)$ is advisable to use a step function or wavelets.

The achieved value of the angular superresolution increases with the increase in the number of functions $N$. The solutions, however, are becoming less and less stable. Errors appear in the form of false sources of signals and in the form of distortions of radio images of real sources.

As a result, for each specific problem there is a certain limiting value of the number of functions $N$, above which the solution obtained becomes unstable. The degree of attainable level of exceeding the Rayleigh criterion depends on the noise level, measurement accuracy, accuracy of setting the characteristics of the measurement system and on the system of functions used.

\subsection{Using a priori information about the source}

It is well known that an increase in the quality of solutions of inverse problems is achieved by using. priori information about the solution. Using this information makes it possible to increase the stability of solutions and, ultimately, to increase the angular superresolution.

In addition to a priori information about the source, the proposed method allows to reveal "hidden", i.e. clearly not specified characteristics of sources. At each step of the iterative process used obtained in the previous step modern data about the source signal characteristics and identify new characteristics.

Search and further use in the iterative process of a priori unknown information about the source is an essential part of the method for solving the inverse problems under consideration.

The dimension of SLAE (3) is limited due to the increasing instability. Therefore, the solutions (2) can be expressed only with the help of a relatively small number of functions $N$. On the basis of the revealed "hidden" and a priori information about the solution, only the most suitable functions for the representation $I(\varphi, \theta)$ are selected from the system of functions $g_{m}(\varphi)$. The selection of functions is an important element in the process of solving a problem. It allows to increase the superresolution without increasing $N$, i.e. without reducing the stability of the solutions.

The analysis of the obtained images, carried out while solving inverse problems, can be effectively used in order to recognize the objects under study.

\subsection{Results and analysis of numerical experiments}

On the mathematical model of a large flat equidistant antenna array, the inverse problems of reconstructing images of objects with superresolution were studied. The source of the signal was set and the inverse problem of obtaining its radio image was then solved in the presence of random components in the processed signals. 
Problem 1. The classical problem of angular resolution was considered. The signal source consisted of two identical point sources on the angular distance between them, less than the width of the DN $\theta_{0.5}$, i.e. not resolved by Rayleigh test.

Initially, the decision was searched without any prior information. The first step was to select the sector $\Omega$ in which the signal source is located. Its boundaries were approximately estimated by reducing $U(\varphi)$ by a factor of two.

As a system of functions $\{g(\varphi)\}$ at the beginning, since there was no information about the source, trigonometric functions were chosen. Further, on the basis of the analysis of the preliminary decisions obtained, the sector of the source angles $\Omega$ was reduced. In agreement with this, the used system of functions $\{g(\varphi)\}$ was also changed.

The obtained preliminary solutions showed that the source under study has significant intensity gradients. This predetermined the use of step functions to continue solving the problem.

The final solution is shown in Fig. 1 in the form of a bold curve. The dashed line is the signal under investigation; the thin solid line is the true source. The resulting solution highlighted two small targets and their location.

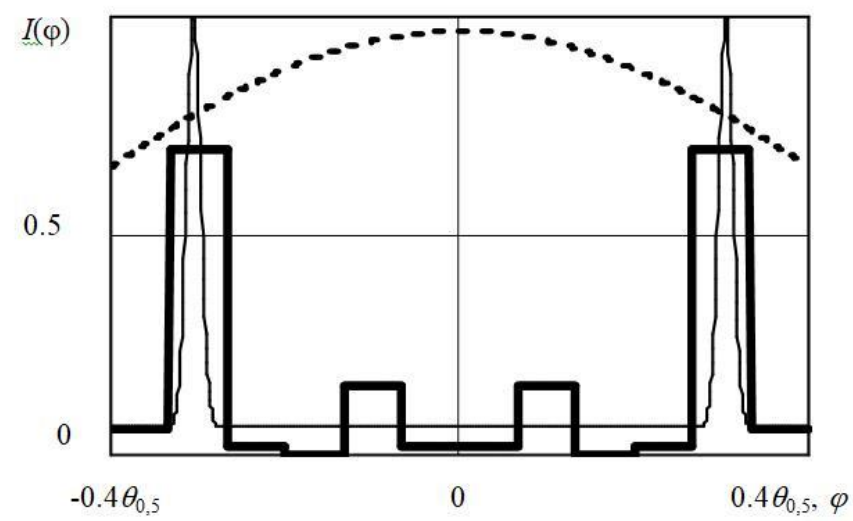

Fig. 1. The reconstructed image of a complex object.

Fig. 2 shows the results of reconstructing a two-dimensional image of three identical small-sized signal sources (thin curve) that are not resolved by direct observation. The grid surface is the signal under study. The obtained approximate solutions are shown in the form of a bold curve. Solutions almost coincided with real source images. Two-dimensional step functions were used as $g_{m}(\varphi, \theta)$.

Figure 3 shows the results of the image reconstruction of two identical small-sized signal sources (thin curve) by identifying the "hidden" characteristics of the source.

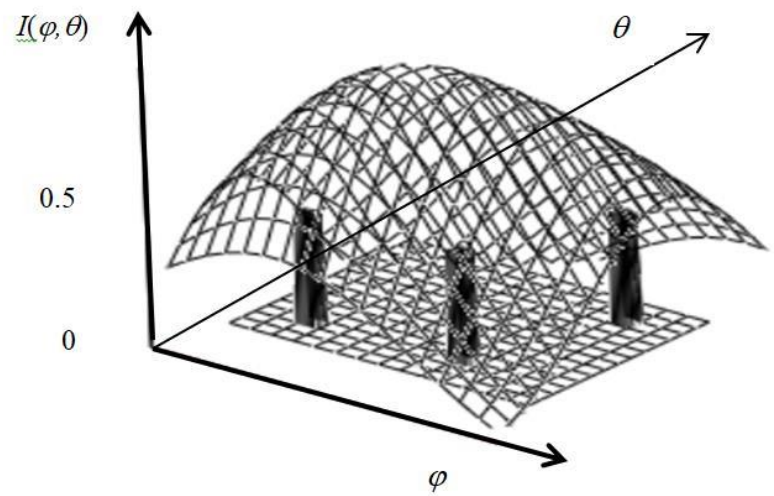

Fig. 2. Reconstructed image of a two-dimensional object. 


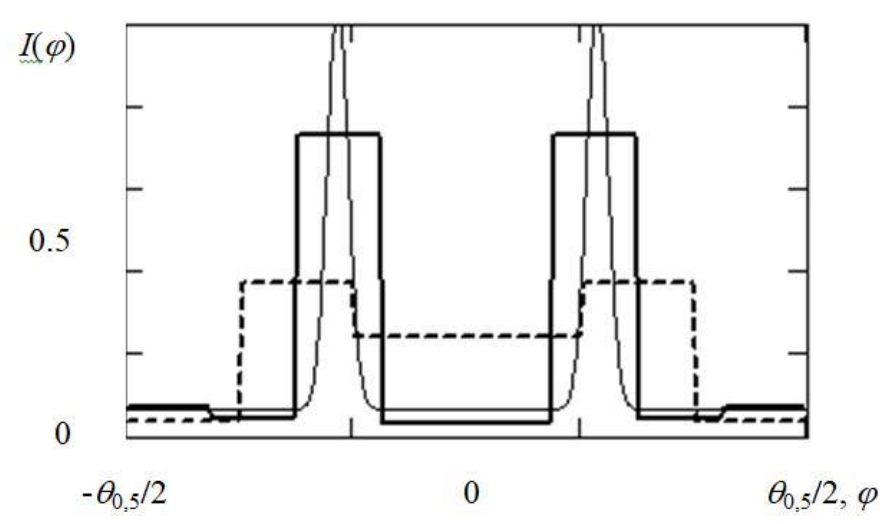

Fig. 3. Reconstructed image of a signal source based on Haar wavelets.

Algorithm based on the use of Eq. (3-6), i.e. without the proposed method of identifying "hidden" characteristics, allowed to resolve the signal sources and restore their images with some error on the angular distance between them up to $\theta_{0,5} / 3$ (dashed line).

The achieved resolution exceeded the Rayleigh criterion three times. In the process of solving using the method of identifying "hidden" characteristics at each step of the iterative process, the area of location of sources was refined. This made it possible to upgrade the system of functions used at each step.

As a result, the obtained stable solution (the solid bold curve) with almost no errors localized the position of the sources with a resolution five times higher than the Rayleigh criterion.

The advantages of the proposed method are particularly evident when there is a high level of noise [12]. Fig. 4 shows the results of source image reconstruction based on the use of MHAT wavelets. The noise level is comparable to the useful signal and was $30 \%$ of the useful signal.

The dotted curve is the real intensity distribution, the solid thin curve is the reconstructed image without the use of data mining methods, the solid bold curve is the image using the iterative process of identifying the "hidden" characteristics of the solution.

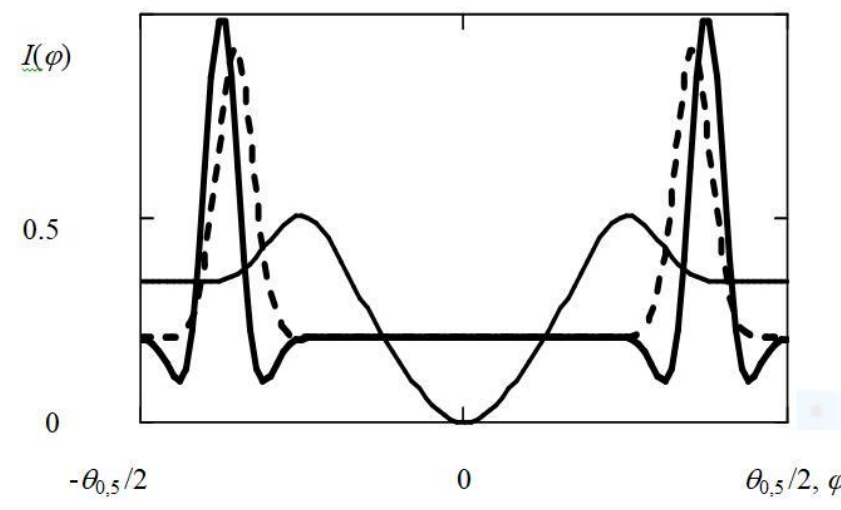

Fig. 4. The reconstructed image of the source using MHAT wavelets.

The resulting solution based on data mining provided an increase in resolution of 3 - 5 times compared with the Rayleigh criterion, the angular accuracy of localization of the source $\theta_{0.5} / 8$. It is necessary to highlight the high noise immunity of the solution, exceeding the known methods of obtaining superresolution. 
Problem 2. A priori, it is known or found in the process of identifying "hidden" information that a signal source is a combination of smooth areas and small-sized sources with a high radiation intensity. In this case, MHAT-wavelets or DOG-wavelets can be effectively used.

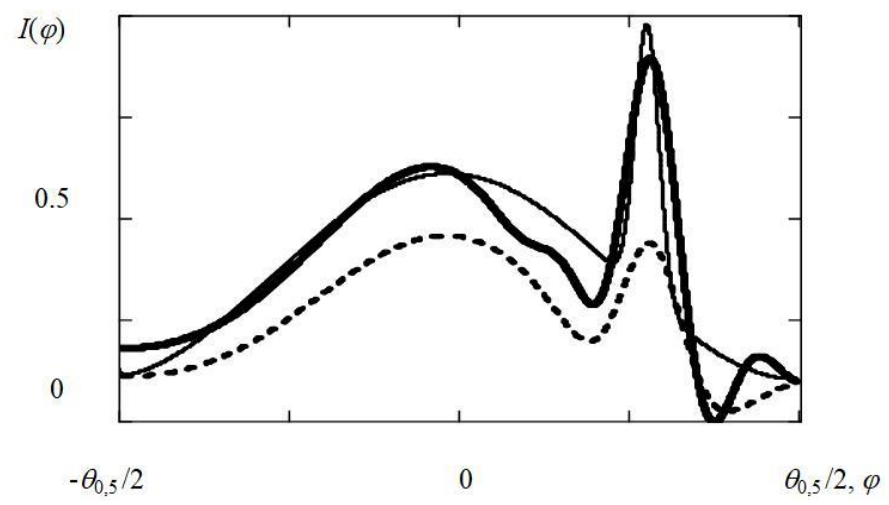

Fig. 5. The reconstructed image of the source using DOG wavelets.

Figure 5 shows the results of source image reconstruction using DOG wavelets. The thin solid line indicates the initial amplitude distribution, the dotted line shows the reconstructed image using 5 wavelets, the bold line displays the reconstructed image using 7 DOG wavelets.

A stable solution with an angular superresolution was obtained, which exceeds the Rayleigh criterion by 5 times and ensures the localization of signal sources with an accuracy of $\theta_{0,5} / 20$. Solutions obtained without the use of a priori information did not allow obtaining an adequate radio image of the source.

Numerical experiments on the model showed that the acceptable $\mathrm{S} / \mathrm{N}$ ratio at the obtained super-resolution level was $15-20 \mathrm{~dB}$. When the Rayleigh criterion is exceeded by 2-3 times, the acceptable $\mathrm{S} / \mathrm{N}$ ratio was $12-14 \mathrm{~dB}$.

The results show that the proposed method makes it possible to restore the radio images of objects with an angular superresolution at lower $\mathrm{S} / \mathrm{N}$ ratio than the conventional methods.

\section{Conclusions}

The developed algebraic signal processing methods make it possible to form radio images of complex one- and two-dimensional objects with superresolution. The achieved angular resolution increases with the use of a priori information about the solution and reaches values 5-10 times higher than the Rayleigh criterion.

Images are restored with relatively small errors in the intensity values. It is confirmed that the degree of exceeding the Rayleigh criterion mainly depends on the signal-to-noise ratio. The results of numerical studies have shown that the minimum $\mathrm{S} / \mathrm{N}$ ratio required for obtaining a sustainable solution with superresolution using a priori information is 12-16 $\mathrm{dB}$. This is significantly less than when using known methods.

The proposed high-speed algorithms allow you to use them in real time.

The reported study was partially supported by RFBR, research project No. 18-57-45001.

Authors are thankful to DST, New Delhi for the financial support (INT/RUS/RFBR/P-341) provided for the present work. 


\section{References}

1. A. Morse, H. Feshbach, Methods of theoretical physics (McGraw-Hill Science/Engineering/Math, 1953)

2. W.U. Bajwa, K. Gedalyahu, Y.C. Eldar, Identification of Parametric Underspread Linear Systems and Super-Resolution Radar, IEEE Transactions on Signal Processing, v. 52, No. 5, pp. 2548-2561 (2011)

3. Y. Zhang, A. Jakobsson, J. Yang, Range-Recursive IAA for Scanning Radar Angular Super-Resolution, EEE Geosci. Remote Sens. Lett., No 14, pp.1675-1679 (2017)

4. B.A. Lagovsky, A.B. Samokhin, Y.V. Shestopalov, Creating Two-Dimensional Images of Objects with High Angular Resolution, IEEE Asia-Pacific Conf. on Antennas and Propagation (APCAP), Conf. Publications, pp. 114 - 115 (2018)

5. B.A. Lagovsky, A.B. Samokhin, Y.V. Shestopalov, Increasing Effective Angular Resolution Measuring Systems Based on Antenna Arrays, Proc.of the 2016 URSI Intern. Symposium on Electromagnetic Theory (EMTS), pp. 432 - 434 (2016)

6. B.A. Lagovsky, A.B. Samokhin, Y.V. Shestopalov, Increasing accuracy of angular measurements using UWB signals, $11^{\text {th }}$ European Conf. on Antennas and Propagation (EUCAP), IEEE Conf. Publications, pp. 1083 - 1086 (2017)

7. B.A. Lagovsky, A.B. Samokhin, Superresolution in signal processing using a priori information, International Conf. Electromagnetics in Advanced Applications (ICEAA), pp. $779-783(2017)$

8. B.A. Lagovsky, Superresolution in signal processing using smart antenna, Progress in Electromagnetics Research Symp. (PIERS), pp. $471-474$ (2017)

9. B.A. Lagovsky, A.B. Samokhin, Y.V. Shestopalov, Creating Two-Dimensional Images of Objects with High Angular Resolution, 2018 IEEE Asia-Pacific Conf. on Antennas and Propagation (APCAP), pp. 114 - 115 (2018)

10. B.A. Lagovsky, A.G. Chikina, Superresolution in signal processing using a priori information, Progress in Electromagnetics Research Symp. (PIERS), pp. 944- 947 (2017)

11. B.A. Lagovsky, A.B. Samokhin, Y.V. Shestopalov, Regression Methods of Obtaining Angular Superresolution, URSI 2019 Asia-Pacific Radio Science Conference (APRASC), IEEE Conf. Paper (2019)

12. G.V. Kulikov, N.V. Dung, Analysis of noise immunity of reception of signals with multiple phase shift keying under the influence of scanning interference, Russian technological journal, v. 6, No. 6, pp. 5-12 (2018) 